\title{
Anthocyanins Participate in Protection of Wheat Seedlings from Osmotic Stress
}

\author{
O.Yu. Shoeva ${ }^{1 *}$, E.I. GordeEva ${ }^{1}$, V.S. Arbuzova ${ }^{1}$ and E.K. KhlestKina ${ }^{1,2}$ \\ ${ }^{1}$ Institute of Cytology and Genetics, Siberian Branch of the Russian Academy of Sciences, \\ Lavrentjeva Ave. 10, Novosibirsk, 630090 Russia \\ ${ }^{2}$ Novosibirsk State University, Pirogova St. 2, Novosibirsk, 630090 Russia \\ (Received 15 February 2016; Accepted 11 August 2016; \\ Communicated by H. Grausgruber)
}

\begin{abstract}
Plant secondary metabolites anthocyanins are considered to play a protective role. In bread wheat (Triticum aestivum L.), anthocyanins can be observed in both adult plants and seedlings. The aim of the current study was to investigate the putative role of anthocyanins present in grains and shoots with respect to the protection of seedlings against drought. For this purpose a set of near isogenic lines (NILs) differing in pericarp and coleoptile colour was used. Water stress was created by artificial shortage of moisture under laboratory conditions. Differences among the lines were observed in a way that the lines with dark-purple grains and coleoptiles (genotype Pp-DIPp-D1Pp3Pp3Rc-A1Rc-A1Rc-D1Rc-DI) demonstrated a higher seedling drought tolerance than plants with uncoloured pericarp and lightpurple coleoptiles ( $p p-D 1 p p-D 1 p p 3 p p 3 R c-A 1 R c-A 1 r c-D 1 r c-D 1)$. Furthermore, protection of the root system and the shoot was related with the presence of anthocyanins in grains and coleoptiles, respectively.
\end{abstract}

Keywords: Triticum aestivum L., flavonoids, near isogenic lines, grain, purple pericarp, red coleoptile

\section{Introduction}

Plants are sessile organisms which are constantly exposed to a variety of biotic and abiotic stresses. To overcome different stress stimuli and their combinations, plants developed mechanisms for multiple stress tolerance, which are activated in contrast to stressspecific protective reactions independently on the type of stress stimuli (Pandey et al. 2015). Anthocyanin biosynthesis is considered as a component of these universal protective reactions against distinct types of stresses (Chalker-Scott 1999).

At least four mechanisms of anthocyanins' plant protective role in severe environments are considered. One is that anthocyanins act as photoprotective light screen, quenching excess photons and, thereby, mitigate photoinhibitory and photo-oxidative damage (Steyn et al. 2002; Merzlyak et al. 2008; Solovchenko and Merzlyak 2008). A further mechanism is based on scavenging free radicals. Due to their high antioxidant

*Corresponding author; E-mail: olesya_ter@bionet.nsc.ru; Phone: +7(383)363-49-63; Fax: +7(383)333-12-78 
capacity anthocyanins neutralize reactive oxygen and nitrogen species compounds almost four times more effectively than ascorbic acid and $\alpha$-tocopherol do (Bors et al. 1994; Wang et al. 1997). Since anthocyanins are present in cells in glycosylated forms they are also considered as osmolytes contributing to depression of osmotic potential and maintenance of turgor pressure during drought-stressed conditions (Chalker-Scott 1999). Moreover, anthocyanins are also able to chelate some heavy metal ions, preventing in such a way oxidative stress (Hale et al. 2001, 2002; Fedenko 2008).

In bread wheat (Triticum aestivum L., BBAADD, $2 n=6 x=42$ ), anthocyanin pigments can be synthesized in different parts of the plant such as coleoptile (controlled by $R c$ genes), culm $(P c)$, leaf blade $(P l b)$, leaf sheath $(P l s)$, glumes $(P g)$, auricle $(R a)$, grain pericarp (complementary genes $P p-1$ and $P p 3$ ), and anthers $(P a n)$. The genes predetermining anthocyanin coloration in wheat have been mapped: $R c, P c, P l b, P l s, P p-1, R a$, $P a n$ form gene clusters on homologous group 7 chromosomes, $P p 3$ and $P g$ map to chromosome 2A (Khlestkina 2013; Khlestkina et al. 2014).

The aim of the current study was to investigate the putative protective role of anthocyanins present in wheat grains and coleoptiles, in particular, to investigate whether they can protect spring wheat seedlings from damaging drought at early growth stages (leaf development, tillering), when drought can cause $13-60 \%$ of wheat yield reduction (Blum et al. 1990; Ivanova et al. 2011).

To investigate this issue a precise genetic model - a set of NILs differing by the allelic state of genes conferring purple grain $(P p)$ and coleoptile $(R c)$ colour was used.

\section{Materials and Methods}

\section{Plant material}

The wheat genotypes used in the study were: (1) 'Saratovskaya 29' ('S29'), carrier of the dominant $R c-A 1$ allele (determining light-purple coleoptiles) and $P p$ alleles conferring uncoloured pericarp ( $p p-D 1 p p-D 1 p p 3 p p 3 P p-A 1 P p-A 1)$; (2) near isogenic lines (NILs) developed in 'S29' background (Arbuzova et al. 1998), but having an additional $R c$ gene $(R c-D 1)$ conferring intensive dark-purple coleoptile pigmentation and two dominant complementary genes $P p-D 1$ and $P p 3$ determining intensive anthocyanin pigmentation of pericarp, inherited from genetic stocks 'Purple Feed' ('PF') and 'Purple' ('P'). These lines, designated i:S29Pp-A1Pp-D1Pp3 ${ }^{\mathrm{PF}}$ ('iPF') and i:S29Pp-A1Pp-D1Pp3 $3^{\mathrm{P}}$ ('iP'), respectively, have been characterized earlier using microsatellite markers, and differ only in the length of the introgressed fragments in the chromosomes 2A and 7D (Tereshchenko et al. 2012; Gordeeva et al. 2015).

\section{Stress treatment}

Osmotic stress was induced by $15 \%$ polyethylene glycol (PEG 6000) according to Yudina et al. (2015). The seeds were placed on filter paper, moistened with distilled water and kept for 48 hours in a RUMED ${ }^{\circledR}$ climate chamber (Rubarth Apparate $\mathrm{GmbH}$ ) at $4{ }^{\circ} \mathrm{C}$ 
without light to synchronize germination. Thereafter the temperature inside the chamber was increased to $20^{\circ} \mathrm{C}$ and a daily cycle with $12 \mathrm{~h}$ light $/ 12 \mathrm{~h}$ darkness was set up. Germinated seeds were exposed to either $15 \%$ or $0 \%$ (control) PEG 6000 solution. All experiments were carried out using seeds produced in the same year and under the same climatic conditions. The experiment was performed in triplicate for each genotype and treatment, with sixteen seedlings per replicate. The root and shoot lengths of each seedling were measured daily from the $3^{\text {rd }}$ to the $7^{\text {th }}$ day after germination.

\section{Anthocyanin extraction and measurement}

For anthocyanin content evaluation, the three NILs were grown at the same conditions as described above. In total 20 seedlings were grown per each replicate and every day from the $3^{\text {rd }}$ to the $6^{\text {th }}$ day after germination, coleoptiles from four seedlings of each NILs were pooled and homogenized in $1 \% \mathrm{HCl} / \mathrm{methanol}$ in the proportion $5 \mathrm{ml}$ of the solution to $1 \mathrm{~g}$ of fresh plant material. The mixture was incubated at $4{ }^{\circ} \mathrm{C}$ for 24 hours and centrifuged at $10,000 \mathrm{~g}$ for $30 \mathrm{~min}$ at $4{ }^{\circ} \mathrm{C}$. A $100 \mu \mathrm{l}$ of the collected supernatant was used for the measurement of the relative anthocyanin content at a wavelength of $530 \mathrm{~nm}$ (OD530) using a SmartSpec ${ }^{\mathrm{TM}}$ Plus spectrophotometer (BioRad).

Anthocyanin content evaluation in pericarp was performed at early or soft dough stage of grain maturity. Pericarp tissue from grains of one spike for each replicate was peeled with a scalpel. The anthocyanin extraction procedure was the same as mentioned above.

\section{Statistical analysis}

The data are presented as mean value \pm standard error $(n=3)$. Significance of differences between treated and control samples of the NILs were assessed using non-parametric Mann-Whitney $U$-test, taking $p \leq 0.05$ as significant. Changes in root length were calculated as follows:

$$
\left(\frac{\text { root length under stress condition }}{\text { root length in control }}-1\right) \times 100 \%
$$

Changes in shoot length and anthocyanin content were calculated accordingly.

The non-parametric Kruskal-Wallis $H$-test was used for determining the influence of the factor 'colour' on changes of growth parameters of wheat seedlings. Two groups of samples were distinguished: one group denominated as 'non-coloured' included 'S29', the second group ('coloured') combined 'iPF' and 'iP'. Spearman's rank correlation coefficients between the parameters were calculated. All statistical analysis was carried out with Statistica v. 6.1 software (StatSoft, Inc.). 


\section{Results}

\section{Shoots and roots length}

The lengths of roots were not differed between 'S29' and 'iPF' or ' $\mathrm{P}$ ' in control and PEGtreated samples, whereas statistically significant differences were observed in the lengths of shoots between the genotypes (Table 1).

Daily osmotic stress led to significantly reduced lengths of shoots and roots in the seedlings (Fig. 1). However, the extent of growth parameters changes varied among the NILs. Reduction in the lengths of shoots and roots was more severe in 'S29' compared to 'iPF' and 'iP'. Shoots length decrease of 'S29' was 18.6 to $28.1 \%$ (on average $24.3 \%$ during all days of the experiment), whereas in ' $\mathrm{iPF}$ ' and ' $\mathrm{iP}$ ', it varied from 11.3 to $19.9 \%$ (16.6\%) and from 11.5 to $20.4 \%(16.6 \%)$, respectively (Fig. 1a). Reduction in the lengths of roots was 10.3 to $22.6 \%(16.0 \%)$ in 'S29', whereas in 'iPF' and 'iP', it varied from 5.4 to $18.4 \%(11.0 \%)$ and from 3.8 to $14.7 \%(8.4 \%)$, respectively (Fig. 1 b).

Table 1. The shoots and roots lengths of 'S29', 'iPF' and 'iP' and relative anthocyanin content in coleoptiles under control and osmotic stress. Values are mean of three replications \pm standard error

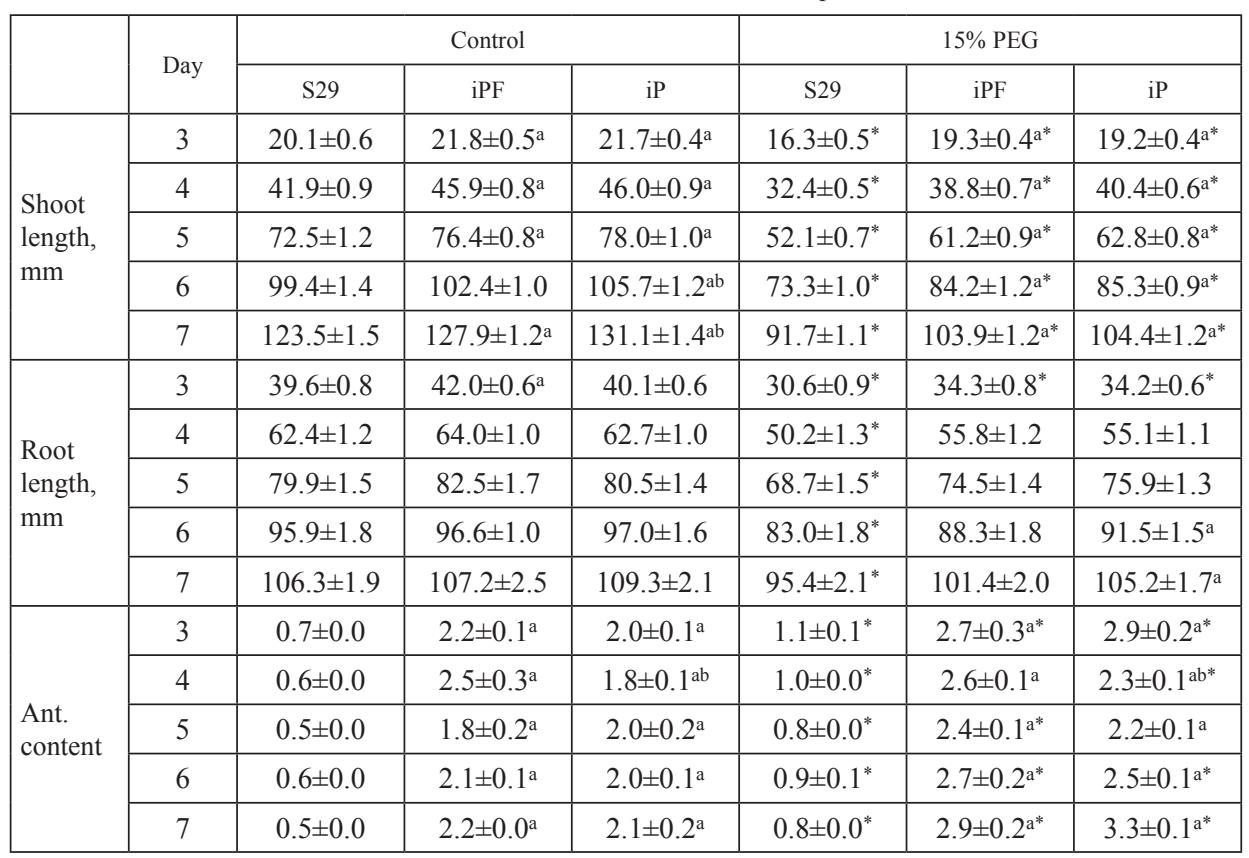

aMean value of 'iPF' or ' $\mathrm{iP}$ ' is significantly different from that one of ' $\mathrm{S} 29$ ' under control or stress condition $(p \leq 0.05$, $U$-test).

'Mean value of 'iP' is significantly different from that one of 'iPF' under control or stress condition ( $p \leq 0.05, U$-test).

*Mean value of stressed sample is significantly different from control one ( $p \leq 0.05, U$-test). 

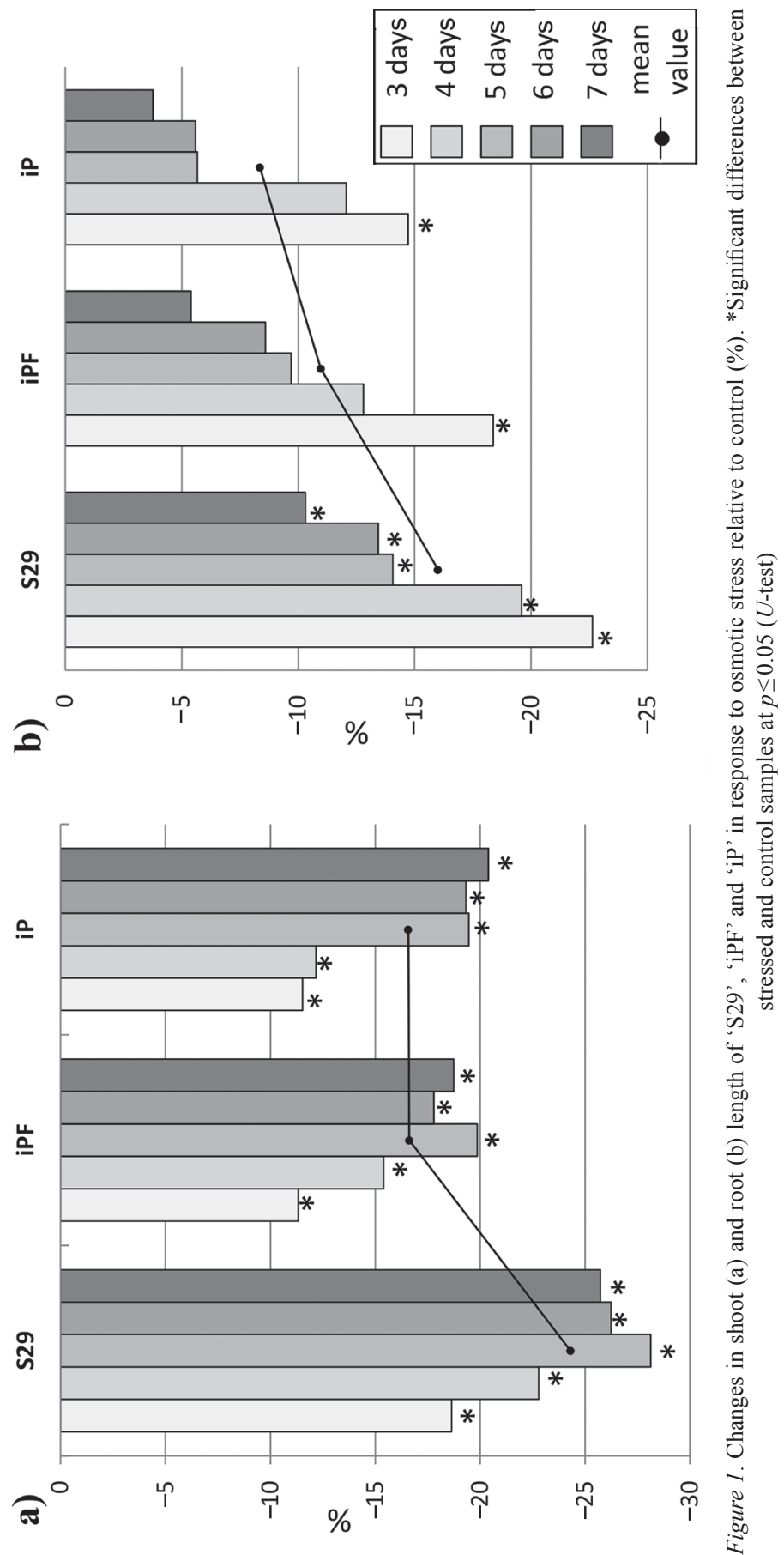

Cereal Research Communications 45, 2017 


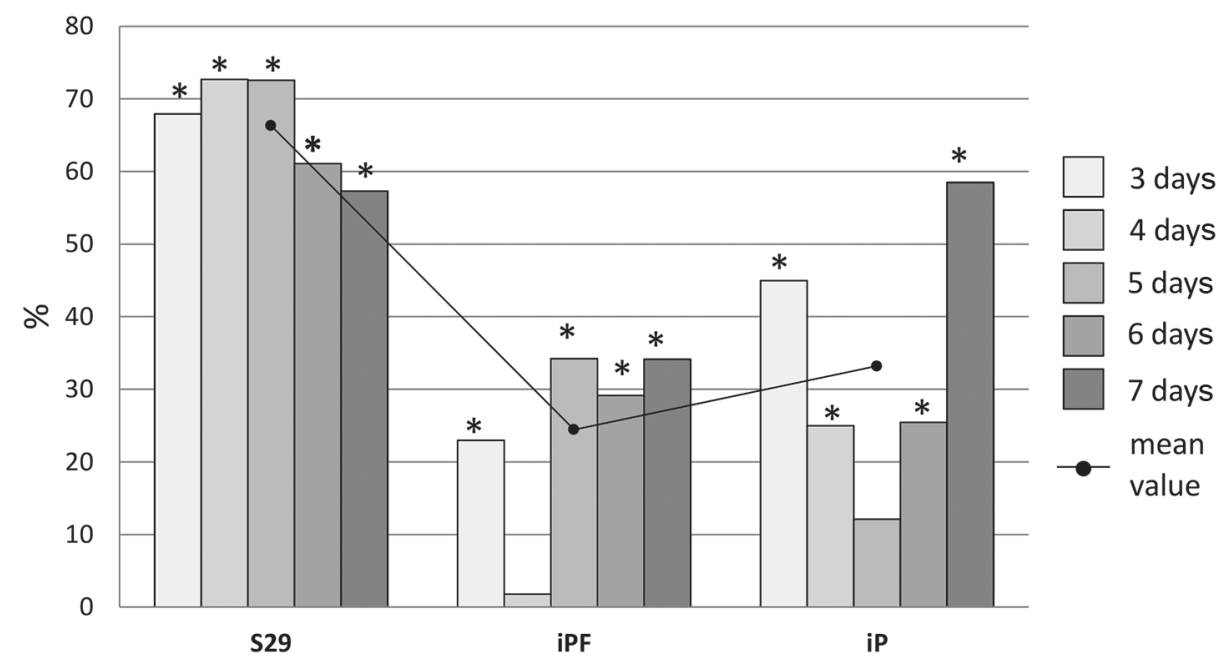

Figure 2. Changes in anthocyanin content in coleoptiles of 'S29', 'iPF' and 'iP' in response to osmotic stress relative to control $(\%)$. Significant differences between stressed and control samples at $p \leq 0.05$ ( $U$-test)

\section{Anthocyanin content}

The NILs differ in anthocyanin content in coleoptiles (Table 1). 'iPF' and 'iP' have more then 3-fold more anthocyanins compared to 'S29'. Anthocyanins were not present in grains of 'S29', whereas their content was significantly different between 'iPF' (2.3 \pm 0.2$)$ and 'iP' (3.5 \pm 0.3$)$ lines.

Osmotic stress increased the concentration of anthocyanins in coleoptiles of all genotypes (Table 1). The intensification of anthocyanin biosynthesis relatively to untreated controls was more pronounced in 'S29' than in the coloured lines 'iPF' and 'iP' (Fig. 2). In 'S29', osmotic stress caused an increase of anthocyanin content in coleoptiles by 57.3$72.7 \%$ (on average $66.3 \%$ during all days of the experiment), whereas in ' $\mathrm{PPF}$ ' and ' $\mathrm{P}$ ' anthocyanin content increased by $1.8-34.2 \%$ (24.5\%) and $12.1-58.5 \%$ (33.2\%), respectively, in comparison with the control.

There was moderate negative correlation between anthocyanin content in coleoptile under control conditions and changing the anthocyanin content in response to osmotic stress $\left(r_{s}=-0.718, p \leq 0.01\right)$. Obtained data demonstrated that osmotic stress causes intensification of the anthocyanin biosynthesis in wheat seedlings with a more pronounced increase in the less coloured genotype 'S29'.

\section{Influence of the coleoptile and grains colour on growth parameters}

The one-way ANOVA on ranks showed that two groups of samples ('non-coloured' vs 'coloured') were significantly different not only in anthocyanin content in coleoptiles and grains under control conditions but also with respect to the changes in anthocyanin con- 
Table 2. Kruskal-Wallis $H$-test analysis. Group size: 'non-coloured' group has five values of traits of 'S29', 'coloured' group has ten values of traits of 'iPF' and 'iP'; degrees of freedom (df): 1

\begin{tabular}{|c|c|c|c|c|}
\hline Trait & Group & Sum of ranks & $H$ & $p$-value \\
\hline \multirow{2}{*}{ Anthocyanin content in coleoptile (in control) } & 'non-coloured' & 15 & \multirow{2}{*}{9.38} & \multirow{2}{*}{0.0022} \\
\hline & 'coloured' & 105 & & \\
\hline \multirow{2}{*}{ Anthocyanin content in grains } & 'non-coloured' & 15 & \multirow{2}{*}{10.5} & \multirow{2}{*}{0.0012} \\
\hline & 'coloured' & 105 & & \\
\hline \multirow{2}{*}{ Changing the anthocyanin content in coleoptile } & 'non-coloured' & 64 & \multirow{2}{*}{8.64} & \multirow{2}{*}{0.0033} \\
\hline & 'coloured' & 56 & & \\
\hline \multirow{2}{*}{ Changing the length of roots } & 'non-colored' & 57 & \multirow{2}{*}{4.34} & \multirow{2}{*}{0.0373} \\
\hline & 'coloured' & 63 & & \\
\hline \multirow{2}{*}{ Changing the length of shoots } & 'non-coloured' & 60 & \multirow{2}{*}{6.00} & \multirow{2}{*}{0.0143} \\
\hline & 'coloured' & 60 & & \\
\hline \multirow{2}{*}{ Root length (in control) } & 'non-coloured' & 35 & \multirow{2}{*}{0.38} & \multirow{2}{*}{0.5403} \\
\hline & 'coloured' & 85 & & \\
\hline \multirow{2}{*}{ Shoot length (in control) } & 'non-coloured' & 35 & \multirow{2}{*}{0.38} & \multirow{2}{*}{0.5403} \\
\hline & 'coloured' & 85 & & \\
\hline
\end{tabular}

tent in coleoptiles and length of roots and shoots under stress conditions (Table 2), whereas no differences between the groups were observed for lengths of roots and shoots under control conditions. The data obtained demonstrated that factor 'dark-purple colour' has a significant impact on changes in growth parameters of wheat seedlings under stress conditions.

\section{Correlation between anthocyanin level and growth parameters}

Moderate negative correlations were observed between anthocyanin content in coleoptiles of control plants and reduction of shoot length under osmotic stress $\left(r_{s}=-0.721\right.$, $p \leq 0.01)$ and between anthocyanin content in grains and reduction of root length under stress $\left(r_{s}=-0.548, p \leq 0.05\right)$.

\section{Discussion}

Protective functions of anthocyanins have been suggested based on an increase of anthocyanins and the expression of related genes under different stress conditions (ChalkerScott 1999; Lo Piero et al. 2005; Castellarin et al. 2007; Nakabayashi et al. 2014; Sanchita et al. 2015). However, some studies have demonstrated that the anthocyanin content is not always increased under stress. The accumulation of anthocyanins in response to stress has been reported to be genotype dependent. Daneshmand et al. (2010) observed in wild po- 
tato species under salinity stress a decrease in anthocyanin content in salt sensitive species and no change in salt-tolerant ones. Ploenlap and Pattanagul (2015) observed anthocyanin overaccumulation under drought stress in a drought-tolerant rice and reduced anthocyanin content in a drought-sensitive variety. Contrary effects (i.e. an increase in a sensitive variety and a decrease in a tolerant variety) were observed under salt stress in tomato (Borghesi et al. 2011). Effects of genes not related to anthocyanin biosynthesis may hinder establishing the role of anthocyanins in stress resistance.

In the current study, using differently coloured wheat NILs the protective functions of anthocyanins under osmotic stress were shown. ANOVA and correlation analysis suggest that anthocyanins protect the wheat seedlings under osmotic stress. These results were obtained for two independent sister lines ('iPF' and 'iP') differing only in the length of the introgressed fragments in chromosomes 2A and 7D carrying the regulatory genes of anthocyanin biosynthesis and the donors of the genes. The investigation revealed that pigments from different organs have a 'local' protective effect: anthocyanins in pericarp improve seedlings' root elongation, whereas anthocyanins in coleoptiles protect shoot elongation under osmotic stress. The protective role of anthocyanins accumulated in the grain pericarp might be related to an effect on seed germination and radical emergence.

Although the NILs used in the current study do not allow addressing the question how anthocyanin biosynthesis is regulated in stress sensitive and stress-tolerant wheat genotypes, it allows the finding of some peculiarities of the biosynthesis under osmotic stress in differently coloured sister lines. PEG treatment induced anthocyanin accumulation in all lines, but in different genotype-dependent manner: higher accumulation relative to the control was observed in the less pigmented 'S29' (Fig. 2). Nonetheless the higher intensification of the anthocyanin synthesis in 'S29' did not lead to its better growth ability (Fig. 1) what allows the conclusion that the protective role of anthocyanins under osmotic stress is predetermined rather by the anthocyanin content in unstressed conditions than by the ability to increase the anthocyanin biosynthesis under stress.

It is also worth noting that the same set of the NILs demonstrated a different pattern of anthocyanins accumulation under cold stress: anthocyanin content decreased in less pigmented 'S29' and increased in 'iPF' and 'iP' (Gordeeva et al. 2013). These data allow the hypothesis of a stress-dependent anthocyanin biosynthesis regulation in the present NILs. Further investigations of the NILs under different types of stresses would be necessary to confirm this hypothesis and might reveal other features of the protective functions of anthocyanins.

\section{Acknowledgements}

We thank Ms Galina Generalova (ICG SB RAS) for technical assistant. Olesya Shoeva thanks RFBR (grant No 16-34-60052) for partial support of this study. Elena Gordeeva, Valentina Arbuzova and Elena Khlestkina thank ICG project (No 0324-2015-0005) for financial support. 


\section{References}

Arbuzova, V.S., Maystrenko, O.I., Popova, O.M. 1998. Development of near-isogenic lines of the common wheat cultivar 'Saratovskaya 29'. Cereal Res. Commun. 26:39-46.

Blum, A., Ramaiah, S., Kanemasu, E.T., Paulsen, G.M. 1990. Wheat recovery from drought stress at the tillering stage of development. Field Crop. Res. 24:67-85.

Borghesi, E., González-Miret, M.L., Escudero-Gilete, M.L., Malorgio, F., Heredia, F.J., Meléndez-Martínez, A.J. 2011. Effects of salinity stress on carotenoids, anthocyanins, and colour of diverse tomato genotypes. J. Agric. Food Chem. 59:11676-11682.

Bors, W., Mochel, C., Saran, M. 1994. Flavonoid antioxidants: Rate constants for reactions with oxygen radicals. Methods Enzymol. 234:420-429.

Castellarin, S.D., Pfeiffer, A., Sivilotti, P., Degan, M., Peterlunger, E., Gaspero, G. 2007. Transcriptional regulation of anthocyanin biosynthesis in ripening fruits of grapevine under seasonal water deficit. Plant Cell Environ. 30:1381-1399.

Chalker-Scott, L. 1999. Environmental significance of anthocyanins in plant stress responses. Photochem. Photobiol. 70:1-9.

Daneshmand, F., Arvin, M.J., Kalantari, K.M. 2010. Physiological responses to NaCl stress in three wild species of potato in vitro. Acta Physiol. Plant. 32:91-101.

Fedenko, V.S. 2008. Tsianidyn yak endohennyy khelator ioniv metaliv u korenyakh prorostkiv kukurudzy [Cyanidin as endogenous chelator of metal ions in maize seedling roots.] Ukr. Biokhim. Zh. (Ukr. Biochem. J.) 80:102-106. [in Ukrainian]

Gordeeva, E.I., Shoeva, O.Y., Khlestkina, E.K. 2013. Cold stress response of wheat genotypes having different $R c$ alleles. Cereal Res. Commun. 41:519-526.

Gordeeva, E.I., Shoeva, O.Y., Khlestkina, E.K. 2015. Marker-assisted development of bread wheat near-isogenic lines carrying various combinations of Pp (purple pericarp) alleles. Euphytica 203:469-476.

Hale, K.L., McGrath, S.P., Lombi, E., Stack, S.M., Terry, N., Pickering, I.J., George, G.N., Pilon-Smits, E.A. 2001. Molybdenum sequestration in Brassica species. A role for anthocyanins? Plant Physiol. 126:13911402.

Hale, K.L., Tufan, H.A., Pickering, I.J., George, G.N., Terry, N., Pilon, M., Pilon-Smits, E.A.H. 2002. Anthocyanins facilitate tungsten accumulation in Brassica. Physiol. Plant. 116:351-358.

Ivanova, G.F., Levitskaya, N.G., Shatalova, O.V. 2011. Vliyanie ekstremalnyih proyavleniy klimaticheskih izmeneniy na produktivnost selskohozyaystvennyih kultur [Influence of extreme displays of climatic changes on efficiency of agricultural crops.] Izvestiya of Saratov University. New series. Series: Earth Sciences 11:41-47. [in Russian]

Khlestkina, E.K. 2013. Genes determining the coloration of different organs in wheat. Russ. J. Genet. Appl. Res. 3:54-65.

Khlestkina, E.K., Gordeeva, E.I., Arbuzova, V.S. 2014. Molecular and functional characterization of wheat near-isogenic line 'i:S29Ra' having intensive anthocyanin pigmentation of the coleoptile, culm, leaves and auricles. Plant Breed. 133:454-458.

Lo Piero, A.R., Puglisi, I., Rapisarda, P., Petrone, G. 2005. Anthocyanins accumulation and related gene expression in red orange fruit induced by low temperature storage. J. Agric. Food Chem. 53:9083-9088.

Merzlyak, M.N., Melø, T.B., Naqvi, K.R. 2008. Effect of anthocyanins, carotenoids and flavonols on chlorophyll fluorescence excitation spectra in apple fruit: signature analysis, assessment, modelling, and relevance to photoprotection. J. Exp. Bot. 59:349-359.

Nakabayashi, R., Mori, T., Saito, K. 2014. Alternation of flavonoid accumulation under drought stress in Arabidopsis thaliana. Plant Signalling Behav. 9:e29518.

Pandey, P., Ramegowda, V., Senthil-Kumar, M. 2015. Shared and unique responses of plants to multiple individual stresses and stress combinations: physiological and molecular mechanisms. Front. Plant Sci. 6:723.

Ploenlap, P., Pattanagul, W. 2015. Effects of exogenous abscisic acid on foliar anthocyanin accumulation and drought tolerance in purple rice. Biologia 70:915-921. 
Sanchita, Singh, R., Mishra, A., Dhawan, S.S., Shirke, P.A., Gupta, M.M., Sharma, A. 2015. Physiological performance, secondary metabolite and expression profiling of genes associated with drought tolerance in Withania somnifera. Protoplasma 252:1439-1450.

Solovchenko, A.E., Merzlyak, M.N. 2008. Screening of visible and UV radiation as a photoprotective mechanism in plants. Russ. J. Plant Physiol. 55:719-737.

Steyn, W.J., Wand, S.J.E., Holcroft, D.M., Jacobs, G. 2002. Anthocyanins in vegetative tissues: A proposed unified function in photoprotection. New Phytol. 155:349-361.

Tereshchenko, O.Y., Gordeeva, E.I., Arbuzova, V.S., Börner, A., Khlestkina, E.K. 2012. The D genome carries a gene determining purple grain colour in wheat. Cereal Res. Commun. 40:334-341.

Wang, H., Cao, G., Prior, R.L. 1997. Oxygen radical absorbing capacity of anthocyanins. J. Agric. Food Chem. 45:304-309.

Yudina, R.S., Leonova, I.N., Salina, E.A., Khlestkina, E.K. 2015. Effect of alien genomic introgressions on the osmotic stress resistance of wheat. Russ. J. Genet. Appl. Res. 5:168-173. 